\title{
Effect of Fin Length and Location on Natural Convection Heat Transfer in a Wavy Cavity
}

\author{
Md. Fayz-Al-Asad ${ }^{1, *}$, M. J. H. Munshi ${ }^{2}$, Md. Manirul Alam Sarker ${ }^{3}$ \\ ${ }^{1}$ Department of Civil Engineering, Dhaka International University, Satarkul, Badda, Dhaka-1212, \\ Bangladesh \\ ${ }^{2}$ Department of Mathematics, Hamdard University Bangladesh, Hamdard Nagar, Gazaria, \\ Munshigonj-1510, Bangladesh \\ ${ }^{1,3}$ Department of Mathematics, Bangladesh University of Engineering and Technology (BUET), \\ Dhaka-1000, Bangladesh
}

Received: 29 March 2020; Received in revised form: 28 June 2020; Accepted: 25 July 2020;

Published online 8 August 2020

(C) Published at www.ijtf.org

\begin{abstract}
The present study aims to analyze the natural convection flow and heat transfer in a wavy cavity with a single horizontal fin attached to its hot wall. Galerkin weighted residual finite element technique has been employed to solve the governing nonlinear dimensionless equations. The effects of model parameters like Rayleigh number, fin length and location on the fluid flow and heat transfer are investigated. The obtained results are exhibited graphically in terms of flow structure, temperature dispersion, velocity field, fin effectiveness, local Nusselt number, and average Nusselt number. It is observed that the different fin length and location have a substantial effect on flow structure and temperature field. Fin effectiveness is also studied and the highest fin effectiveness was found at fin length $(L=$ 0.75). Besides, it is also found that the mean Nusselt number increases significantly with the increase of Rayleigh number and fin length. Wavy cavity becomes more effective on heat transfer behaviors and fluid flow than that of a square cavity.
\end{abstract}

Keywords: Finite Element Method, Wavy Cavity, Natural convection, and Fin.

\section{Introduction}

Natural convection heat and mass transfer inside an enclosure have received considerable attention due to its implication in various engineering application like geophysics, drying technologies, geothermal reservoirs, crude oil production, heat exchangers and so on.

A good number of researches have been done on different shapes of cavities. Bilgen [1] investigated numerically natural convection in cavities with a thin fin on the hot wall. It was found that the heat transfer rate was a lower limit with the fin attached to the middle of

*Corresponding e-mail: fayzmath.buet@gmail.com (Md. Fayz-Al-Asad) 
Md. Fayz-Al-Asad et al.

International Journal of Thermofluid Science and Technology (2020), Volume 7, Issue 3, Paper No. 070303

near the middle of the heated wall. Shi and Khodadadi [2] numerically analyzed the steady laminar natural convection heat transfer in a differentially heated square cavity due to a thin fin on the hot wall. They found that for a bigger value of Rayleigh number, the rate of heat transfer was increased an irrespective of the fin location or length.

\begin{tabular}{|c|c|c|}
\hline \\
\hline \multicolumn{3}{|c|}{$\begin{array}{l}\text { Nomenclature } \\
A \quad \text { amplitude }\end{array}$} \\
\hline$C_{P}$ & specific heat, $\mathrm{J} \mathrm{kg}^{-1} \mathrm{~K}^{-1}$ & $\alpha \quad$ thermal diffusivity, $\mathrm{m}^{2} \mathrm{~s}^{-1}$ \\
\hline$B$ & dimensionless fin thickness, $b / W$ & thermal expansion coefficient, $\mathrm{K}^{-1}$ \\
\hline$g$ & gravitational acceleration, $\mathrm{ms}^{-2}$ & number of oscillations \\
\hline$h^{*}$ & heat transfer coefficient & density of the fluid, $\mathrm{kg} \mathrm{m}^{-3}$ \\
\hline$H$ & dimensionless fin position, $h / W$ & dimensionless temperature \\
\hline$L$ & dimensionless fin length, $l / W$ & kinematic viscosity, $\mathrm{m}^{2} \mathrm{~s}^{-1}$ \\
\hline$k$ & thermal conductivity, $W \cdot \mathrm{m}^{-1} \cdot \mathrm{K}^{-1}$ & dynamic viscosity, $\mathrm{kg} \mathrm{m}^{-1} \mathrm{~s}^{-1}$ \\
\hline$N u_{a v}$ & average Nusselt number & effectiveness \\
\hline$N u_{L}$ & local Nusselt number & \\
\hline$N$ & $\begin{array}{l}\text { dimensionless distance normal to } S \\
\text { coordinates, } n / W\end{array}$ & Subscripts \\
\hline $\operatorname{Pr}$ & Prandlt number, $v / \alpha$ & average \\
\hline$R a$ & Rayleigh number, $g \beta\left(\mathrm{T}_{h}-\mathrm{T}_{c}\right) W^{3} / \alpha v$ & cooled \\
\hline$S$ & $\begin{array}{l}\text { dimensionless special coordinate } \\
\text { along enclosure surface }\end{array}$ & $\begin{array}{ll}h & \text { heated } \\
f & \text { fin }\end{array}$ \\
\hline$U, V$ & $\begin{array}{l}\text { dimensionless velocity in } X \text { and } Y \\
\text { direction respectively }\end{array}$ & \\
\hline$W$ & Enclosure height and width & \\
\hline
\end{tabular}

Ben-Nakhi and Chamkha [3] studied numerically conjugate natural convection in a square enclosure with inclined thin fin of arbitrary length. They conclude that increasing the fin length enhanced the mean Nusselt number on cold wall.Tasnim and Collins [4] numerically investigated natural convection heat transfer in a square cavity with a baffle on hot wall. They observed that the effect of fin location on the rate of heat transfer was depended on strongly affected by Rayleigh number, and the fin length. Asad et al. [5] explored numerically investigation of natural convection flow in a hexagonal enclosure having vertical fin. They conclude that the rate of heat transfer enhanced for greater Rayleigh number when fin length is kept constant. Manjunath et al. [6] studied heat transfer analysis on peristaltic transport of Jeffery fluid in an inclined elastic tube with porous walls. They found that an increase in Biot number reduces the magnitude of the temperature. Xu et al. [7] reported the effect of fins and their height on natural convection flow transition cavity. They found that the flow near the finned wall changes from a steady to periodic unsteady flow at a critical Rayleigh number that is sensitive to the fin length. Gdhaidh et al. [8] analytically investigated the enhancement of natural convection heat transfer within closed enclosure using parallel fins. They observed that the maximum heat transfer decreases with the increases of the fin number. Goud et al. [9] studied the mass transfer effects on MHD flow through porous medium past exponential accelerated inclined plate with variable temperature and thermal radiation. Asad et al. [10] numerically investigated MHD mixed convection heat transfer having vertical fin in a lid-driven square cavity. They observed that the heat transfer rate is 
Md. Fayz-Al-Asad et al.

International Journal of Thermofluid Science and Technology (2020), Volume 7, Issue 3, Paper No. 070303

enhanced for greater Richardson number with lower Hartmann number for particular fin length. Alsabery et al. [11] reported the effect of rotating solid cylinder on entropy generation and convective heat transfer in a wavy porous cavity heated from below. They found that an augmenting in the porosity of the medium causes an increase in heat transfer from the wall to the fluid and therefore an increase in the convective flow and consequently a decrease in the Bejan number. Elatar et al. [12] performed a numerical study on laminar natural convection inside square enclosure with a single horizontal fin attached to the hot wall. They discovered the effect of Rayleigh number, fin lengths and fin positions on the flow structure and heat transfer behaviors. Sertel and Bilen [13] reported the effect of using sinusoidal profile in fins on thermal performance. They found that the heat transfer augmentation can be provided for wavy triangular fins at higher amplitude lengths. Shahriari and Ashorynejad [14] numerically analyzed the MHD natural convection of hybrid nanofluid in an open wavy cavity. They showed that the Nusselt number decreases with the increase of the Hartmann number, but it increases by the increament of Rayleigh number and nanoparticle volume fraction. Sheremet and Pop [15] numerically analyzed the natural convection in a wavy porous cavity with sinusoidal temperature distributions on both side walls filled with a nanofluid: Buongiorno's mathematical model. Menni et al. [16] studied numerical analysis of heat and nanofluid mass transfer in a channel with detached and attached baffle plates. They observed that the presence of nanoparticles and baffle plates has significant effects of the rate of heat transfer. Recently Munshi et al. [17] studied MHD free convection heat transfer having vertical fin in a square wavy cavity. They found that, the heat transfer rate is enhanced for greater Rayleigh number with lower Hartmann number with a constant fin length.

Based on the above literature survey, it appears that the study of natural convection heat transfer in a wavy cavity with a single horizontal fin has not yet been studied. Therefore natural convection heat transfer in a wavy cavity with a single horizontal fin will be numerically analyzed in this present study.

\section{Formulation of the Problem and Governing Equations}

\subsection{Problem configuration}

In the present study, we consider a laminar, incompressible two-dimensional natural convection flow in a square wavy cavity of height and width $W$. The geometry and coordinate systems are schematically shown in Fig. 1.

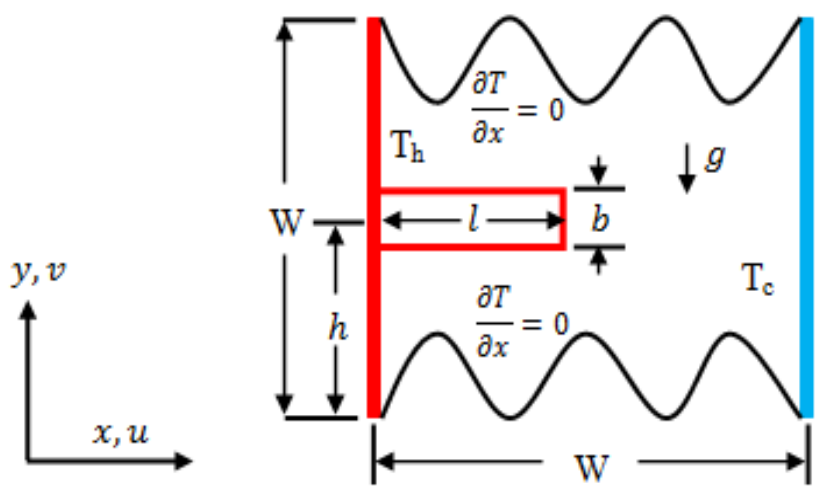

Fig. 1. Schematic description of the physical model with boundary conditions 
Md. Fayz-Al-Asad et al.

International Journal of Thermofluid Science and Technology (2020), Volume 7, Issue 3, Paper No. 070303

The bottom and upper wavy walls are insulated. The left side wall is uniformly heated temperature $T_{h}$ while the right side walls at uniform cooled temperature $T_{c}\left(T_{h}>T_{c}\right)$. A uniformly heated fin of the thickness $(b)$ and length $(l)$ is attached to its left heated wall at a position $(h)$ from the bottom wall.

\subsection{Mathematical Modeling}

Within the framework of the aforementioned assumptions, the governing equations for the present study are expressed in dimensional form as follows:

$$
\begin{aligned}
& \frac{\partial u}{\partial x}+\frac{\partial v}{\partial y}=0 \\
& \rho\left(u \frac{\partial u}{\partial x}+v \frac{\partial u}{\partial y}\right)=-\frac{\partial p}{\partial x}+\mu \nabla^{2} u \\
& \rho\left(u \frac{\partial v}{\partial x}+v \frac{\partial v}{\partial y}\right)=-\frac{\partial p}{\partial y}+\mu \nabla^{2} v+\rho g \beta\left(T-T_{c}\right) \\
& u \frac{\partial T}{\partial x}+v \frac{\partial T}{\partial y}=\alpha \nabla^{2} T
\end{aligned}
$$

Boundary conditions: The boundary conditions for the above-stated model are as follows:

On the left wall: $u=v=0, T=T_{h}$

On the right wall: $u=v=0, T=T_{c}$

On the bottom wavy wall: $u=v=\frac{\partial T}{\partial x}=0 ; A(1-\cos (2 \pi \lambda x))$

On the top wavy wall: $u=v=\frac{\partial T}{\partial x}=0 ; 1-A(1-\cos (2 \pi \lambda x))$

For fin surface: $0 \leq x \leq l ; u=v=0, T=T_{h} ; y=h+\frac{b}{2}$ and $y=h-\frac{b}{2}$

Using the following transformation of variables, the governing equations can be converted to the dimensionless forms:

$$
X=\frac{x}{W}, Y=\frac{y}{W}, U=\frac{u W}{\alpha}, V=\frac{v W}{\alpha}, P=\frac{p W^{2}}{\rho \alpha^{2}}, \theta=\frac{T-T_{c}}{T_{h}-T_{c}}, H=\frac{h}{W}, L=\frac{l}{W} \text { and } B=\frac{b}{W}
$$

where $U$ and $V$ are the velocity components in the $X$ and $Y$-directions respectively, $P$ is the dimensionless pressure and $\theta$ is the dimensionless temperature. After substitution of the transformation of variables into equations (1) - (4), the governing dimensional equations can be written in the following dimensionless form: 


$$
\begin{gathered}
\frac{\partial U}{\partial X}+\frac{\partial U}{\partial Y}=0 \\
U \frac{\partial U}{\partial X}+V \frac{\partial U}{\partial Y}=-\frac{\partial P}{\partial X}+\operatorname{Pr} \nabla^{2} U \\
U \frac{\partial V}{\partial X}+V \frac{\partial V}{\partial Y}=-\frac{\partial P}{\partial Y}+\operatorname{Pr} \nabla^{2} V+\operatorname{Ra} \operatorname{Pr} \theta \\
U \frac{\partial U}{\partial X}+V \frac{\partial U}{\partial Y}=\nabla^{2} \theta
\end{gathered}
$$

The controlling parameters of the governing equations are Prandtl number $(P r)$ and Rayleigh number $(R a)$, which are respectively defined as:

$$
\operatorname{Pr}=\frac{v}{\alpha}, \alpha=\frac{k}{\rho C_{p}} \text { and } R a=\frac{g \beta\left(\mathrm{T}_{h}-\mathrm{T}_{c}\right) W^{3}}{\alpha v}
$$

The transformed dimensionless boundary conditions are as follows:

On the left wall: $U=V=0, \theta=1$

On the top wavy wall: $U=V=\frac{\partial \theta}{\partial X}=0 ; 1-A(1-\cos (2 \pi \lambda X))$

On the right wall: $U=V=0, \theta=0$

On the bottom wavy wall: $U=V=\frac{\partial \theta}{\partial X}=0 ; A(1-\cos (2 \pi \lambda X))$

For fin surface: $U=V=0, \theta=1,0 \leq X \leq L ; Y=H+\frac{B}{2}$ and $B=H-\frac{B}{2}$

Nusselt Number: Firstly, the rate of heat transfer due to convection is defined as:

$$
h^{*} \Delta T=-k \frac{\partial T}{\partial n}
$$

where $n$ denotes the coordinate direction normal to surface. The local Nusselt number $\left(N u_{L}\right)$ and the average Nusselt number $\left(N u_{a v}\right)$ of the cavity can be calculated from the following expression:

$$
N u_{L}=-\left.\frac{\partial \theta}{\partial N}\right|_{\text {surface }} \text { and } N u_{a v}=-\left.\int_{2+2 L}^{3+2 L} \frac{\partial \theta}{\partial N}\right|_{\text {surface }} d s
$$

\section{Computational Procedure}

The governing dimensionless equations (6) - (9) together with the boundary conditions (11a) - (11e) are solved numerically using Galerkin weighted residual finite element method. The details of this method are well described by Taylor and Hood [18] and Dechaumphai [19]. In a nutshell, the method first discretized the solution domain into a finite number of grids, which are composed of non-uniform triangular elements. We used six node 
Md. Fayz-Al-Asad et al.

International Journal of Thermofluid Science and Technology (2020), Volume 7, Issue 3, Paper No. 070303

triangular elements for the development of the finite element equations. After that, the nonlinear governing partial differential equations are transformed into a system of integral equations by applying the Galerkin weighted residual technique. The integration involved in each term of those equations is performed by using the Gauss's quadrature method. Then non-linear algebraic equations so obtained are modified by the imposition of boundary conditions. To solve the set of the global nonlinear algebraic equations in the form of a matrix, the Newton-Raphson iteration technique is used. Finally, these linear equations have been solved by using triangular factorization method. The convergence criterion for the computational procedure along with error estimation has been defined as $\left|\psi^{m+1}-\psi^{m}\right| \leq 10^{-6}$, where $m$ is the number of iteration and is a function of variable $U, V$ and $\theta$.

\section{Grid Refinement Test}

In order to check a proper grid size for the present study at $\operatorname{Pr}=1.42, \operatorname{Ra}=10^{5}$, $L=0.50$ and $H=0.50$. A grid refinement test was performed with five types of meshes; the average Nusselt number of the hot wall is calculated. Table 1 displays the average Nusselt number of the hot wall for several grids. It can be observed from the table, the grid size of 12083 nodes and 23202 elements allowed an acceptable solution for the present studied.

Table 1: Grid refinement test at $\operatorname{Pr}=1.42, R a=10^{5}, L=0.50$ and $H=0.50$

\begin{tabular}{|c|c|c|}
\hline Nodes (elements) & $N u_{a v}$ & Time (s) \\
\hline $1988(3637)$ & 5.0567 & 12 \\
\hline $4739(8850)$ & 5.0690 & 18 \\
\hline $5698(10772)$ & 5.0795 & 24 \\
\hline $12083(23202)$ & 5.0951 & 27 \\
\hline $34799(68190)$ & 5.0973 & 53 \\
\hline
\end{tabular}

\section{Code Validation}

To verify the accuracy of the present numerical code, we have compared our statistical results with Elatar et al. [12] considering, $R a=10^{6}, L=0.20$ and $H=0.50$. Table 2 shows the comparison of the average Nusselt number along the right cold wall. The results show an excellent agreement and promote confidence in using the present numerical code.

Table 2: Comparison of average Nusselt number on the cooled wall for $R a=10^{6}, L=0.20$ and $H=0.50$.

\begin{tabular}{|c|c|c|c|}
\hline$B$ & 0.02 & 0.04 & 0.1 \\
\hline Elatar et al. $[12]$ & 8.672 & 8.710 & 8.947 \\
\hline Present result & 8.685 & 8.721 & 8.965 \\
\hline
\end{tabular}

\section{Results and Discussion}

For this part of the study, we represent numerical simulations are depicted to show the effects of different Rayleigh number $\left(10^{3} \leq R a \leq 10^{6}\right)$ dimensionless fin length $(0.25 \leq L \leq$ 
Md. Fayz-Al-Asad et al.

International Journal of Thermofluid Science and Technology (2020), Volume 7, Issue 3, Paper No. 070303

$0.75)$ and dimensionless fin location $(0.25 \leq H \leq 0.75)$. The values of the Prandtl number, fin thickness, amplitude and number of oscillations are fixed at $\operatorname{Pr}=1.42, B=0.02, A=0.08$ and $\lambda=3$ respectively. A set of graphical results are shows an evolution of streamlines in Figure 2 and isotherms contours in Figure 3, velocity field in Figure 4, local Nusselt number in Figure 5, fin effectiveness in Figure 6 and average Nusselt number in Figure 7.

Figure 2 and Figure 3 show an evolution of streamlines and isotherms contours for $R a$ $=10^{5}, H(0.25,0.50$ and 0.75$)$ and $L(0.25,0.50$ and 0.75$)$. Figure 3 shows that the fin is blocking the eddy upper and bottom sides of the fin for $H=0.25$ and 0.75 respectively. It is also observed that as fin length $(L=0.25,0.50$ and 0.75$)$ increases, the eddy is damped due to the blockage effect for all fin positions. It is clearly depicted from Figure 2 that the fin blockage effect is performing a significant role in the flow structure inside the wavy enclosure.

Figure 3 presents the isotherms contours for $R a=10^{5}$. It can be seen from the figure that the areas of low isotherm lines are the areas of minimum heat transfer. For the fin position, $H=0.75$, isotherms in the upper side along the cooled wall are thick which means higher heat transfer in this region. The increase of the fin lengths $(L=0.50$ and 0.75$)$ and for positions $(H=0.50$ and 0.75$)$ decreases the isotherm lines in the area upper side of the fin, which means lower heat transfer in this region.
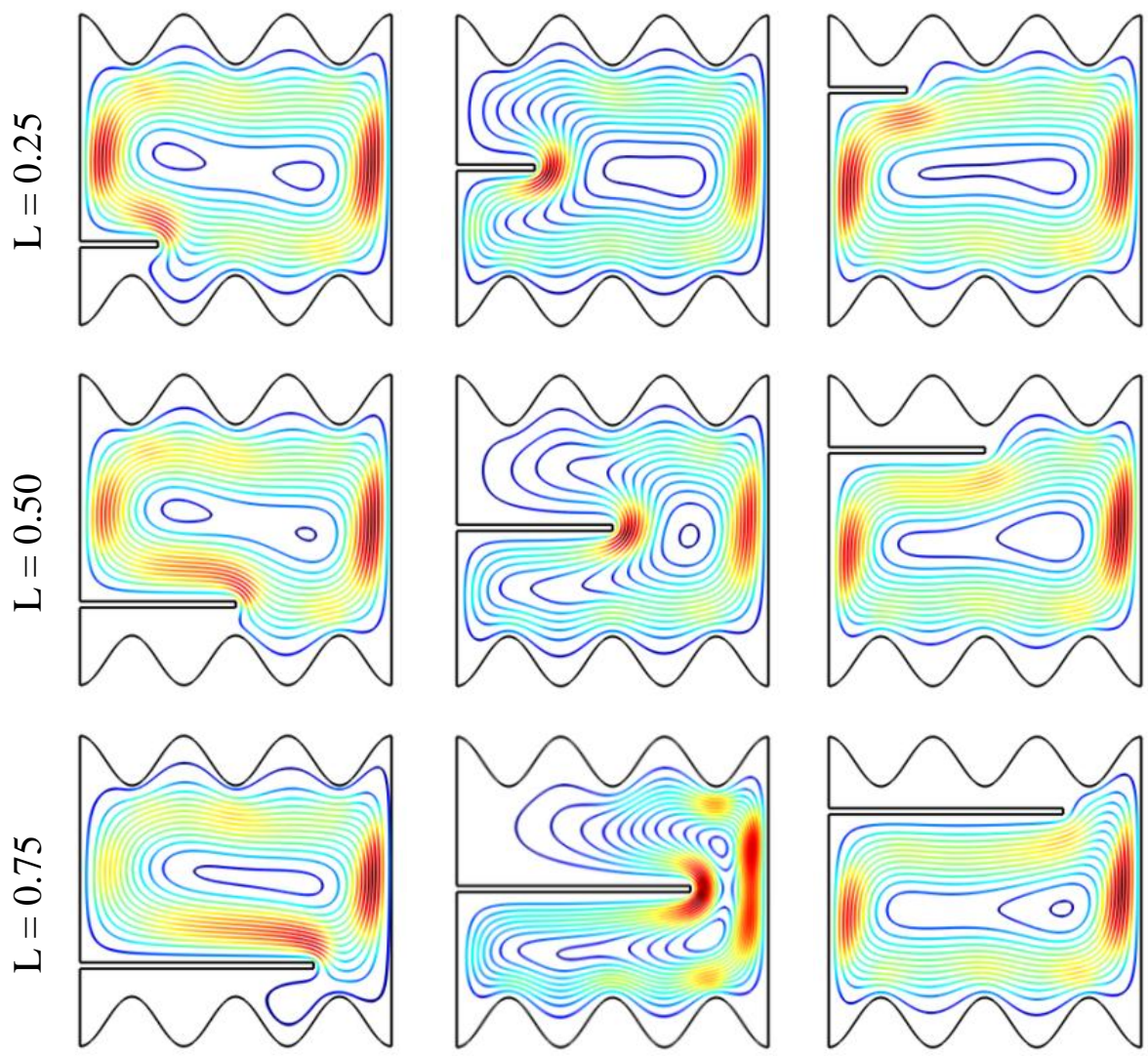

$$
H=0.25
$$

$$
H=0.50
$$

$$
H=0.75
$$

Fig. 2 Variations of the streamlines for various $L$ and $H$ at $R a=10^{5}$ 
Md. Fayz-Al-Asad et al.

International Journal of Thermofluid Science and Technology (2020), Volume 7, Issue 3, Paper No. 070303
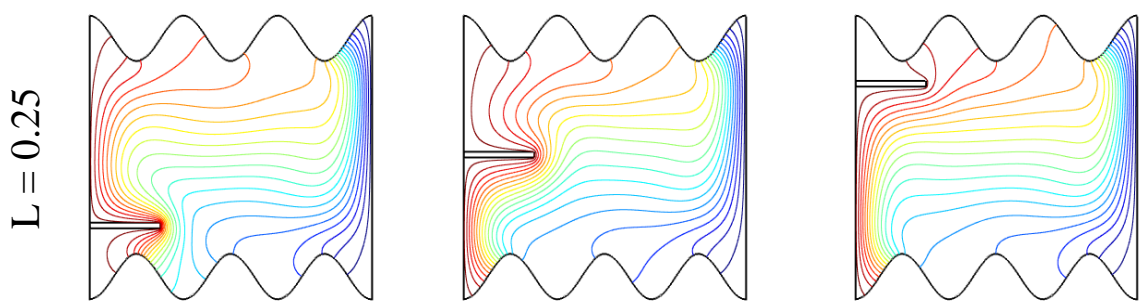

1

0.95

0.89
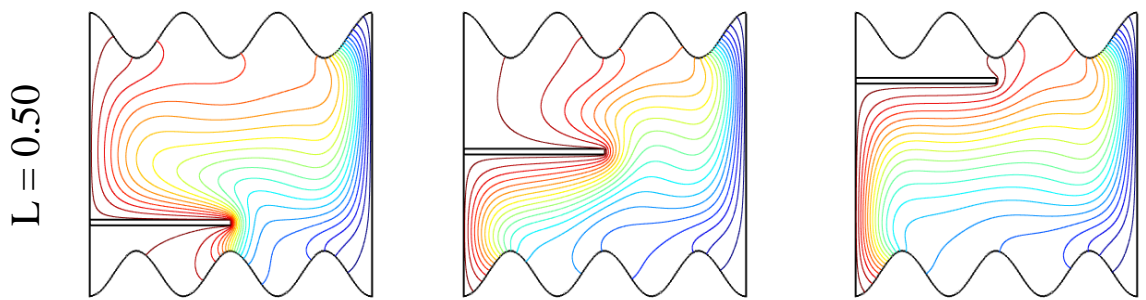

0.84

0.79

0.73

0.68

0.62

0.57

0.52

0.46
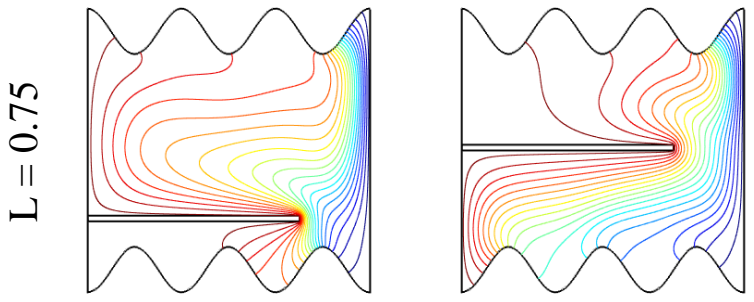

$H=0.50$

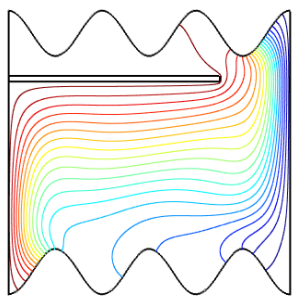

0.41

0.35

0.3

0.25

0.19

0.14

0.09

0.03

$H=0.75$

Fig. 3 Variation of the isotherms for various $L$ and $H$ at $R a=10^{5}$
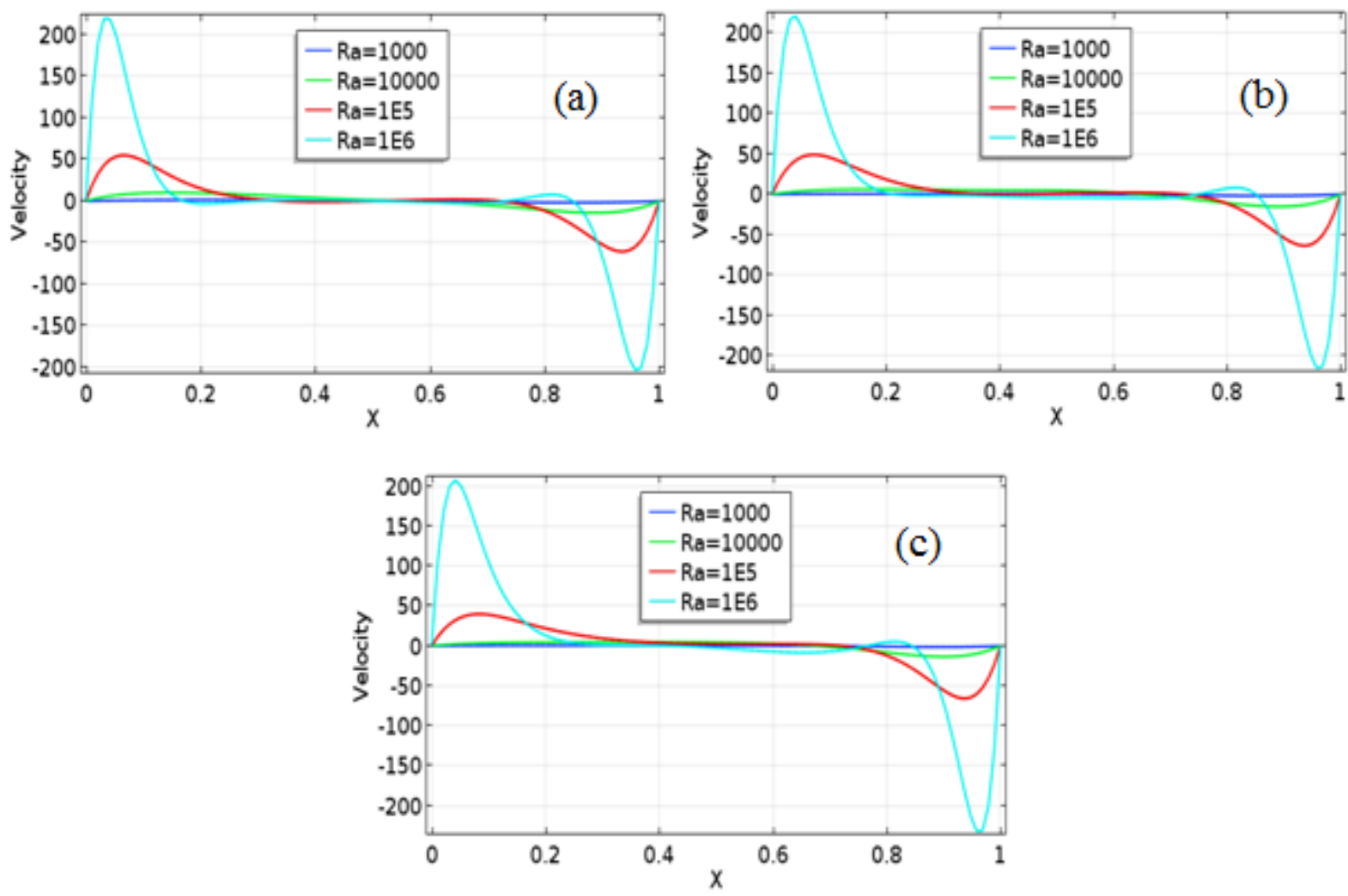

Fig. 4 Variations of velocity filed for (a) $L=0.25$, (b) $L=0.50$, (c) $L=0.75$ at $H=0.25$ 
Md. Fayz-Al-Asad et al.

International Journal of Thermofluid Science and Technology (2020), Volume 7, Issue 3, Paper No. 070303

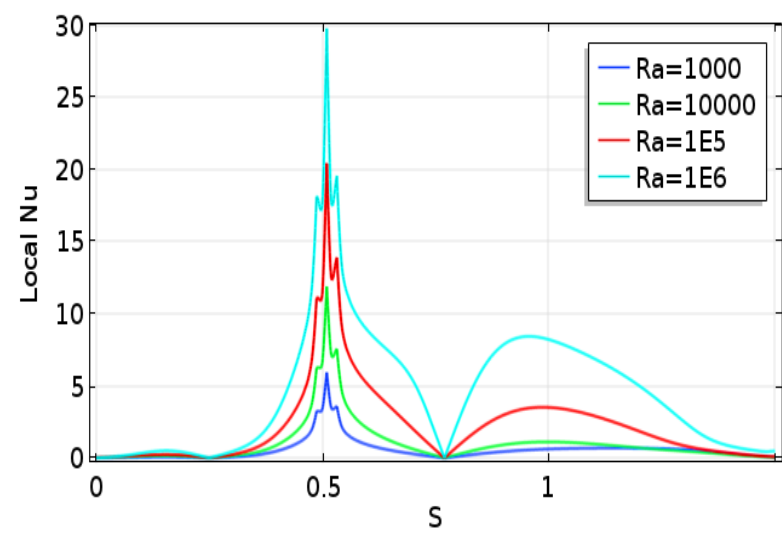

(a) $H=0.25$

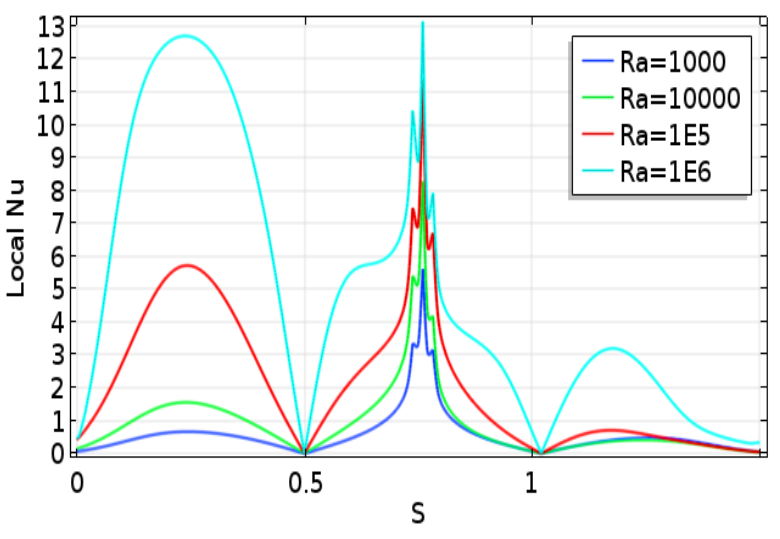

(b) $H=0.50$

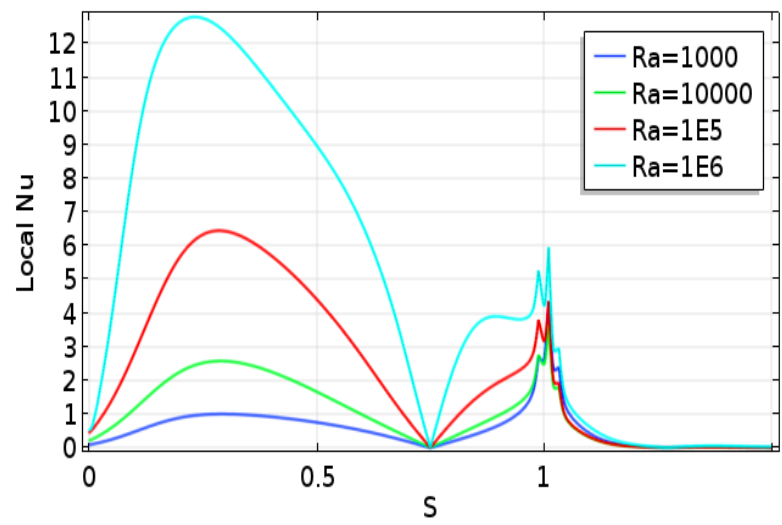

(c) $H=0.75$

Fig. 5 Variations of local Nusselt number for different $R a$ and $H$ at $L=0.25$.

Figure 4 presents the effect of vertical velocity component along the horizontal centre line of an enclosure for different dimensionless fin length $(L)$ and Rayleigh number ( $R a$ ) with dimensionless fin position $H=0.25$ and $P r=1.42$ of the wavy enclosure. It can be observed that the changing rate of velocity is almost similar for $R a=10^{3}$ and $R a=10^{4}$ but one concave up and down for $R a=10^{5}$ and $R a=10^{6}$.

Figure 5 presents the effect of local Nusselt number on the heated part of the left wall and fin surfaces for different dimensionless fin position $(H)$ and Rayleigh number $(R a)$ with dimensionless fin length $L=0.25$ and $\operatorname{Pr}=1.42$ of the wavy enclosure. The changing rate of the local Nusselt number is almost similar for every $R a$. It is clearly depicted from Figure 5 that the minimum and maximum values of the local Nusselt number are found at fin tip position and the top side of the fin.

Fin effectiveness is the parameter that quantifies the heat transfer enhancement inside the cavity with a fin compared to the case with no fin as presented by Elatar et al. [12]

$$
\varepsilon_{f}=\frac{\text { heat transfer with fin }}{\text { heat transfer without fin }}
$$




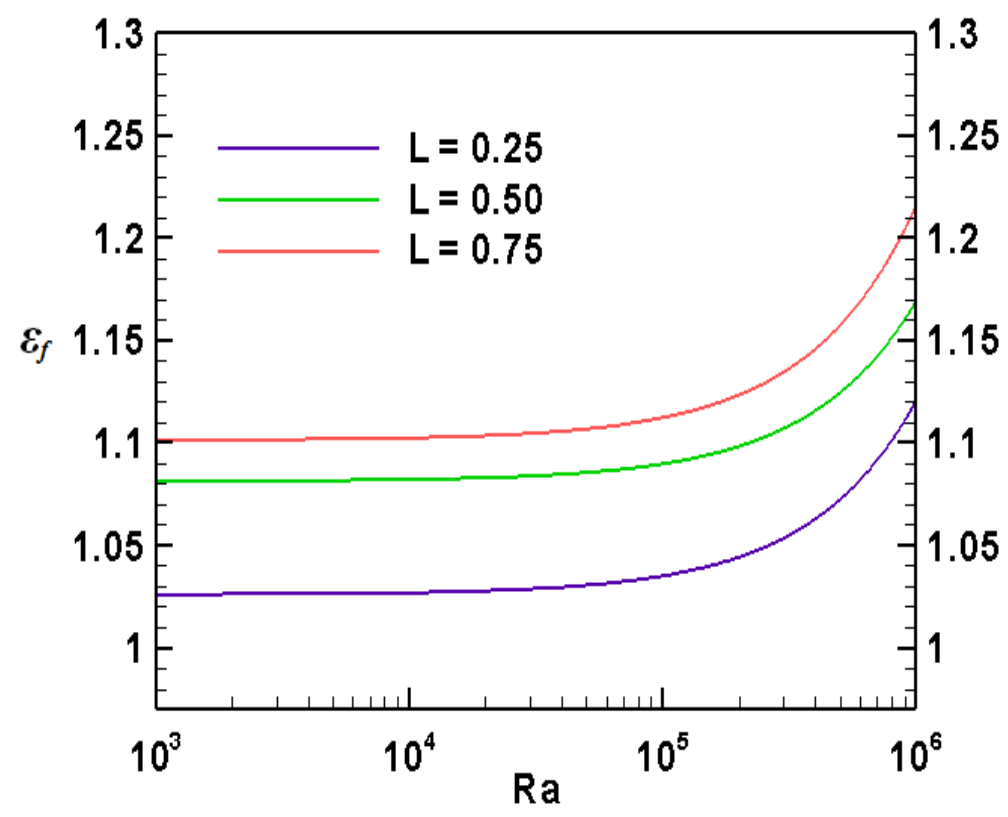

Fig. 6 Variation of fin effectiveness with $R a$ for different fin length $(L)$ at $H=0.50$.

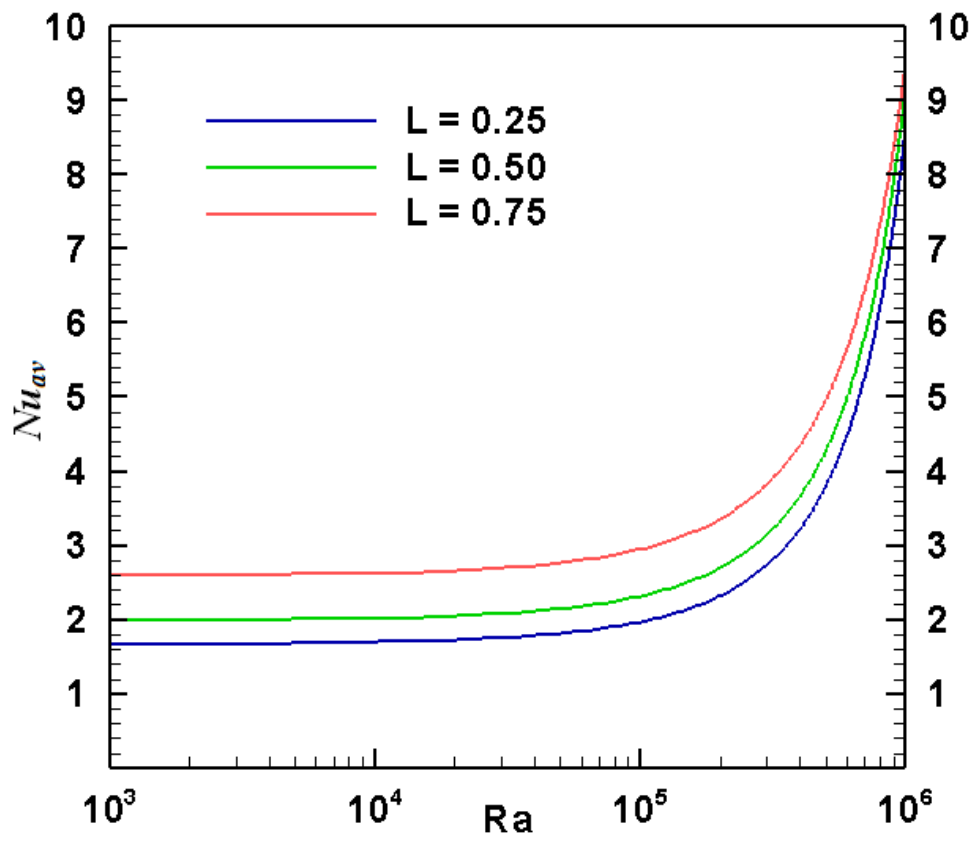

Fig. 7 Variation of average Nusselt number with $R a$ for different dimensionless fin length $(L)$ at $\operatorname{Pr}=1.42, H=0.50$ and $\lambda=3$.

Figure 6 presents the evolution of the fin effectiveness with Rayleigh number $(R a)$ for different dimensionless fin length $(L)$ at $\operatorname{Pr}=1.42, H=0.50$ and $\lambda=3$. It is clearly depicted from Figure 6 that the fin effectiveness increases with increasing the fin length. It is also found that the effectiveness increases with increasing $R a$ for a particular value of fin length.

Figure 7 presents the effect of the average Nusslet number with $R a$ along the right cooled wall for different dimensionless fin length $(L)$ at $\operatorname{Pr}=1.42, H=0.50$ and $\lambda=3$. It is 
Md. Fayz-Al-Asad et al.

International Journal of Thermofluid Science and Technology (2020), Volume 7, Issue 3, Paper No. 070303

clearly shown from Figure 7 that the average Nusselt number increases with increasing the fin length $(L)$ and Rayleigh number $(R a)$.

\section{Conclusion}

In this study, the effect of fin length and position on natural convection heat transfer in a wavy enclosure with a horizontal fin has been performed. Comparisons with the previously published work were performed which gave excellent agreement among the results. In the view of the acquired outcomes, the following findings are listed:

- Fin length and location have a substantial effect on the flow and heat transfer characteristics inside the wavy cavity.

- Velocity field and local Nusselt number increase with increasing Rayleigh number $(R a)$.

- Fin effectiveness is enhanced with increasing of the fin length $(L)$ and Rayleigh number $(R a)$. The highest fin effectiveness is found at $L=0.75$.

- The mean Nusselt number increases significantly with the increase of the Rayleigh number $(R a)$ and fin length $(L)$. The best result is found at $R a=10^{6}$ and $L=0.75$.

In view of these arguments, the present study can be applied in various engineering applications including radiators in cars, computer CPU heat sinks, hydrogen fuel cells, solar collector, and heat exchanging devices and so on.

Acknowledgements: The authors are very grateful and express their sincere thanks to the editor, reviewers, and the team of Journal.

\section{References}

[1] E. Bilgen, Natural convection in cavities with a thin fin on the hot wall, Int. J. Heat and Mass Transf. 48 (2005) 3493-3505.

[2] X. Shi, J. M. Khodadadi, Laminar natural convection heat transfers in a differentially heated square cavity due to a thin fin on the hot wall, J. Heat Transf. 125 (2003) 624-634.

[3] A. Ben-Nakhi, A. J. Chamkha, Conjugate natural convection in a square enclosure with inclined thin fin of arbitrary length, Int. J. Therm. Sci. 46 (2007) 467-478.

[4] S. H. Tasnim, M. R. Collins, Numerical analysis of heat transfer in a square cavity with a baffle on hot wall, Int. Comm. Heat Mass Transf. 31 (2004) 639-650.

[5] M. F. A. Asad, M. M. A. Sarker, M. J. H. Munshi, Numerical investigation of natural convection flow in a hexagonal enclosure having vertical fin, J. Sci. Res. 11 (2019) 173-183.

[6] G. Manjunath, C. Rajashekhar, H. Vaidya, K. V. Prasad, Saraswati, B.B. Divya, Heat transfer analysis on peristaltic transport of Jeffery fluid in an inclined elastic tube with porous walls, Int. J. Thermofluid Sci. Tech. 7 (2020) 20070101.

[7] F. Xu, J. C. Patterson, C. Lei, Effect of fin length on natural convection flow transition in a cavity, Int. J. Therm. Sci. 70 (2013) 92-101. 
Md. Fayz-Al-Asad et al.

International Journal of Thermofluid Science and Technology (2020), Volume 7, Issue 3, Paper No. 070303

[8] F. A. Gdhaidh, K. Hussain, H. S. Qi, Enhancement of natural convection heat transfer within closed enclosure using parallel fins, Int. J. Mechanical Aerospace Industrial and Mechatronics Eng. 9 (2015) 355-360.

[9] B. S. Goud, B. S. Babu, M. N. R. Sheker, G. Srinivas, Mass transfer effects on MHD flow through porous medium past exponential accelerated inclined plate with variable temperature and thermal radiation, Int. J. Thermofluid Sci. Tech. 6 (2019) 19060402.

[10] M. F. A. Asad, M. A. Hossain, M. M. A. Sarker, Numerical investigation of MHD mixed convection heat transfer having vertical fin in a lid-driven square cavity, AIP Conf. Proc. 2121 (2019) 030023-6; https://doi.org/10.1063/1.5115868

[11] A. I. Alsabery, T. Tayebi, A. J. Chamkha, I. Hashim, Effect of rotating solid cylinder on entropy generation and convective heat transfer in a wavy porous cavity heated from below, Int. Comm. Heat Mass Transf. 95 (2018) 197-209.

[12] A. Elatar, M. A. Teamah, M. A. Hassab, Numerical study of laminar natural convection inside square enclosure with single horizontal fin, Int. J. Therm. Sci. 99 (2016) 41-51.

[13] H. Sertel, K. Bilen, The effect of using sinusoidal profile in fins on thermal performance. Int. J. Heat and Tech. 37 (2019) 541-750.

[14] H. R. Ashorynejad, A. Shahriari, MHD natural convection of hybrid nanofluid in an open wavy cavity, Results in Physics, 9 (2018) 440-455.

[15] M. A. Sheremet, I. Pop, Natural convection in a wavy porous cavity with sinusoidal temperature distributions on both side walls filled with a nanofluid: Buongiorno's mathematical model, J. Heat Transf. 137 (2015) 072601-8.

[16] Y. Menni, A. J. Chamkha, C. Zidani, B. Benyoucef, Numerical analysis of heat and nanofluid mass transfer in a channel with detached and attached baffle plates, Mathematical Modeling of Eng. Problems, 6 (2019) 52-60.

[17] M. J. H. Munshi, M. F. A. Asad, R. K. Bhowmik, M. M. A. Sarker, MHD free convection heat transfer having vertical fin in a square wavy cavity, Int. J. Stat. Appl. Math. 4 (2019) 32-38.

[18] C. Taylor, P. Hood, A numerical solution of the Navier-Stokes equations using finite element technique. Computers and Fluids, 1 (1973) 73-89.

[19] P. Dechaumphai, Finite Element Method in Engineering, $2^{\text {nd }}$ ed. Chulalongkorn University Press, Bangkok (1999). 